\title{
Equivalent Circuit of a Magnetic Sensor Coil and a Simple Filter for Rejection of $60 \mathrm{~Hz}$ Man-Made Noise
}

\author{
Liana F. ZAMBResky and Tomiya WATANABE \\ Department of Geophysics and Astronomy, \\ University of British Columbia, \\ Vancouver, British Columbia, Canada
}

(Received August 29, 1977; Revised April 9, 1979)

\begin{abstract}
A magnetic sensor coil is shown to behave as a 2 nd order Butterworth low pass filter for induced e. m. f. if the coil's output ends are terminated with a set of parallel resistor and capacitor matched to the sensor. The conditions for matching are theoretically derived, assuming an equivalent circuit of the sensor coil. An experiment to check the Butterworth filter behavior agrees well with the theory. It demonstrates the validity of the assumed equivalent circuit of the sensor. The corner frequency of the filter can be changed, selecting different matched values of the resistor and capacitor. In the experiment, the corner frequency is set at $6 \mathrm{~Hz}$ which rejects $60 \mathrm{~Hz}$ man-made noise $40 \mathrm{db}$ ca. without distorting magnetic pulsation signals up to the highest frequency range (Pc 1). This technique for rejection of man-made noise is more advantageous than another common practice, putting a twin- $T$ filter between the sensor and the head amplifier. A twin-T filter used this way is shown to give rise to ringing depending on the characteristics of the sensor, the twin-T filter and the input impedance of the head amplifier.
\end{abstract}

\section{Introduction}

Induction magnetometers are used most commonly for observation of magnetic pulsations, quasi-sinusoidal natural magnetic field variations in a frequency range approximately from 0.001 to several $\mathrm{Hz}$. Extremely high sensitivity coils have to be used as sensors, since it is necessary to detect field changes of the order of $0.01 \mathrm{r} / \mathrm{sec}$. Consequently, man-made noise, especially $60 \mathrm{~Hz}$ noise from commercial power lines, creates a serious obstacle for operating induction magnetometers. The main purpose of this paper is to present an effective method to reduce $60 \mathrm{~Hz}$ man-made noise. The method is technically easy and yet scientifically interesting. It provides us with a proof that a sensor coil is electrically equivalent to a simple circuit.

The technique is to terminate the sensor coil with a resistor and capacitor, parallel to each other, whose values are matched with the electrical constants of the 
coil in a manner to be described later. It is then proved that the whole sensor system behaves as a Butterworth filter of the second order for electromotive forces generated in the coil by time-changing magnetic fields. The corner frequency of the Butterworth filter approximately equals the ressonance frequency determined by the selfinductance of the sensor coil and the value of the terminating capacitor. The selfinductance of a sensor coil is usually so high that the corner frequency can be made equal to several $\mathrm{Hz}$, using a capacitor of the order of a few microfarads. Accordingly, attenuation of $60 \mathrm{~Hz}$ noise, $40 \mathrm{db}$ or even more, can be achieved without much difficulty.

\section{Theoretical Consideration}

A sensor coil with a terminating resistor and a capacitor is electrically equivalent to the circuit shown in Fig. 1 . In this figure, $R, L$, and $C$ are the d.c. resistance, selfinductance and capacitance of the sensor coil. The capacitance effect of the coil windings, noted as early as 1910 from general antenna use, is equivalent to that of a

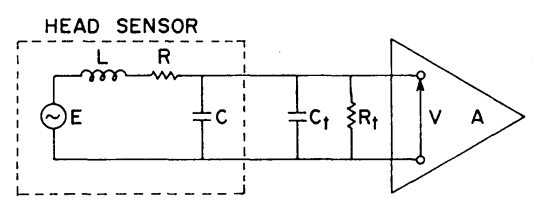

Fig. 1. Equivalent circuit of a magnetic sensor coil. The terminating capacitor and resistor, $C_{\mathrm{t}}$ and $R_{\mathrm{t}}$, with appropriate values make the whole sensor system behave as a Butterworth filter in frequency response.

capacitor parallel to the resistance and self-inductance of the coil (CAMPBELL, 1969). The values of the terminating resistor and capacitor are represented by $R_{\mathrm{t}}$ and $C_{\mathrm{t}}$. If a sinusoidal electromotive force with a frequency $f$ and an amplitude $E_{0}$ is generated in the coil, the amplitude of the voltage output across the terminating resistance is given as follows,

$$
V_{0}=\frac{E_{0}}{1+\alpha} \sqrt{1+\left(\beta^{2}+\alpha^{2} / \beta^{2}-2\right)(\bar{f} / \bar{c})^{2}+\left(f / f_{c}\right)^{4}},
$$

where the notations $\alpha, \beta$, and $f_{\mathrm{c}}$ are defined by

$$
\begin{gathered}
\alpha=R / R_{\mathrm{t}}, \\
\beta=1 / Q=R \sqrt{\left(C+C_{\mathrm{t}}\right) / L},
\end{gathered}
$$

and

$$
1 / f_{\mathrm{c}}=2 \pi \sqrt{L\left(C+C_{\mathrm{t}}\right)} / \sqrt{ } 1+\alpha .
$$

The parameters $\alpha$ and $\beta$ introduced above are non-dimensional. The parameter $\alpha$ 
represents the ratio between the resistance of the sensor coil and that of the terminating resistance. The parameter $\beta$ is the inverse of the $Q$ value of an $R-L-\left(C+C_{\mathrm{t}}\right)$ series circuit. The quantity $f_{\mathrm{c}}$ is equal to the resonance frequency determined by $L$ and $\left(C+C_{\mathrm{t}}\right)$, except for the last factor on the right-hand side of Eq. (4). The voltage output $V$ lags in phase behind the electromotive force $E$ by an angle $\theta$ given as follows,

$$
\theta=\tan ^{-1}\left\{\frac{(1 / \sqrt{1+\alpha})(\beta+\alpha / \beta)\left(f / f_{\mathrm{c}}\right)}{1-\left(f / f_{\mathrm{c}}\right)^{2}}\right\}
$$

Reference is given to UEDA and WATANABE (1975a) concerning the derivation of Eqs. (1) through to (5). Equation (1) shows that the sensor behaves as a Butterworth filter of the second order if the coefficient of the second term inside the square root sign vanishes;

$$
\beta^{2}+\alpha^{2} / \beta^{2}-2=0 .
$$

The corner frequency is then equal to $f_{\mathrm{c}}$ defined by Eq. (4). Ueda and Watanabe (1975a) have shown that there is no $\alpha$ satisfying Eq. (6) if $\beta>\sqrt{2}$. They have shown also that there is no $\beta$ to satisfy the equation if $\alpha>1$. If $0<\alpha<1$, however, there are two values of $\beta$ satisfying Eq. (6),

$$
\beta=\sqrt{1+\sqrt{1-\alpha^{2}}},
$$

and

$$
\beta=\sqrt{1-\sqrt{1-\alpha^{2}}} .
$$

\section{Experiment}

The present authors carried out ar experiment to demonstrate the preceding theory. The sensor used is a solenoidal coil having a high- $\mu$ metal core. The coil is $14^{\prime \prime}$ long and is made of a copper wire. The total turn number is 50,000 . The core is made of laminated and insulated high- $\mu$ metal strips which were cased into a cylindrical polyester tube. The tube's inner diameter is approximately $1^{\prime \prime}$ and the length of the core is $3^{\prime}$. The circuit constants of the coil are as follows,

$$
R=2.23 \mathrm{k} \Omega, \quad L=1.05 \mathrm{kH}, \quad \text { and } \quad C=0.048 \mathrm{nF} .
$$

The inductance and capacitance were measured by means of the anti-resonance method (CAMPBELL, 1969) and several other methods described by UEDA and WATANABE (1975b). The coil was terminated with a resistor $R_{\mathrm{t}}=28 \mathrm{k} \Omega$ and a capacitor $C_{\mathrm{t}}=0.67 \mu \mathrm{F}$. With these choices, the parameters $\alpha$ and $\beta$ become equal to 0.0796 and 0.0565 , respectively (see Eqs. (1) and (2)). They satisfy Eq. (8), and accordingly Eq. (6), viz., the Butterworth condition. The corner frequency $f_{\mathrm{c}}$ is found 
to be $6 \mathrm{~Hz}$, using Eq. (4). A test of the theory was carried out, putting the sensor coil in a calibration coil which creates in itself a uniform and sinusoidal magnetic field. The calibration coil is cylindrical in shape, approximately $8^{\prime}$ in length and $6.5^{\prime \prime}$ in diameter. Its total turn number is 1,000 . The magnetic field created in it is uniform within $\pm 4 \%$, except within a foot of each end. The average field strength, except near the ends, is approximately $520 \gamma$ for a coil current of $1 \mathrm{~mA}$. The current fed into the calibration coil in the experiment was $1 \mathrm{~mA}$ rms. Frequency was scanned from $0.1 \mathrm{~Hz}$ to a few tens of $\mathrm{Hz}$. The output voltage across the terminating resistance was measured with changing frequency. It was then divided by frequency and plotted versus frequency (see Fig. 2). This makes comparison between the theory and the experiment easy, since the electromotive force $E_{0}$ generated in the coil is proportional to frequency. Agreement between the theory and the experiment is excellent. And, it shows that the equivalent circuit shown in Fig. 1 is appropriate at least for frequencies relevant to magnetic pulsations.

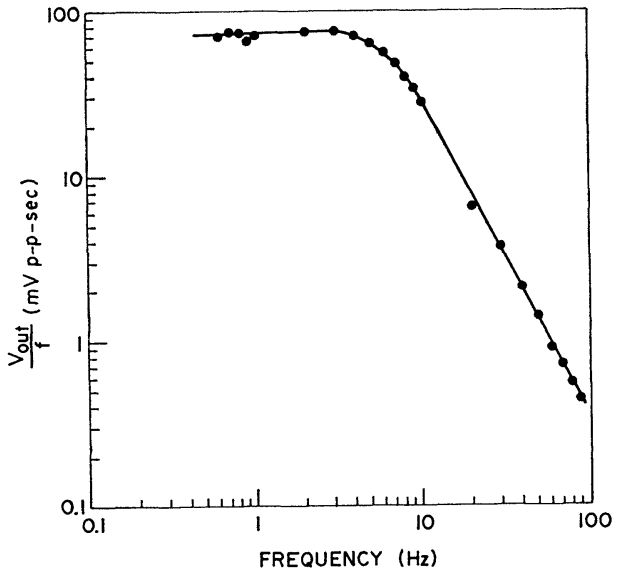

4. Twin-T versus Butterworth Filters
Fig. 2. Frequency response of a sensor with terminating capacitor and resistor. The values of the terminating capacitor and resistor were so chosen as to satisfy Eq. (6).

Twin-T filters are used widely for rejection of $60 \mathrm{~Hz}$ man-made noise. Since twin-T filters are notch filters, they appear to be ideal for rejecting noise at a constant frequency without much distorting the signals at other frequencies. Often, a twin-T filter is placed between the sensor coil and the preamplifier as shown in Fig. 3. With this arrangement, however, the twin-T filter does not necessarily behave as expected. The theory of a twin-T filter as a notch filter is based on the following two assumptions; (i) that the signal source impedance is zero and (ii) that output from the filter is received with an infinitely large impedance. In practice, it is hard to satisfy both conditions. Concerning the signal source impedance, the d.c. resistance of the sensor used for this experiment is $2.23 \mathrm{k} \Omega$. If one wants to reduce the resistance, a thicker 
wire has to be used which makes the sensor heavier. Reactance of the sensor is also significant. It becomes larger than the d.c. resistance for frequencies higher than $\sim 0.34 \mathrm{~Hz}$. The inductance of a sensor cannot be made smaller without reducing the sensitivity. With regard to the impedance on the output side of the twin-T filter, viz., $R_{\mathrm{g}}$, it is equal to the input impedance of the preamplifier if the twin-T filter is connected directly to the amplifier. If a terminating resistance is connected in series with the preamplifier, the impedance on the output side is increased, but the effective gain of the preamplifier is reduced. If a terminating resistance is put parallel to the preamplifier, the impedance on the output side becomes smaller than the input impedance of the preamplifier. In any case, the receiving impedance on the output side cannot be much different from the input impedance of the preamplifier. As a preamplifier for an induction magnetometer, a d.c. chopper amplifier is used most often, since its d.c. stability is superior to that of any other type of amplifier. However, its input impedance is relatively low. Typically, it is in a range from $1 \mathrm{k} \Omega$ to several tens of kilo ohms. It is not too large compared to the d.c. resistance of a typical sensor. In order that the first condition about the theory of twin- $T$ filters be satisfied, the resistance of the filter $R_{1}$ (see Fig. 3) should be much larger than the

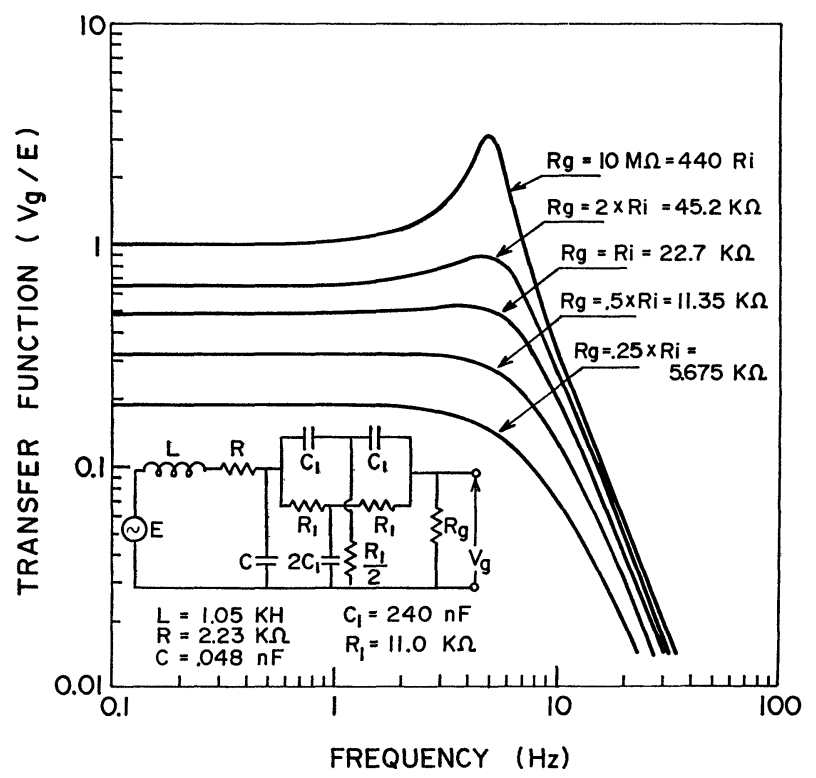

Fig. 3. Frequency response of a sensor plus a twin-T filter system. The twin-T filter rejects a very narrow frequency band centered at $60 \mathrm{~Hz}$ in the ideal case that the source impedance is zero and the output is received with infinite resistance. Being connected with a magnetic sensor having a large value of self-inductance, the twin-T filter does not act as an ideal notch filter. Its behavior greatly depends on the terminating resistance, $R_{\mathrm{g}}$. 
sensor's d.c. resistance $R$. With such a high value of $R_{1}$, however, it becomes difficult to satisfy the second condition about the theory of twin-T filters.

To see more in detail how the twin-T filter arrangement affects performance of an induction magnetometer, a computer simulation for a model system represented by Fig. 3 has been carried out. The self-inductance $L$, the d.c. resistance $R$, and the capacitance $C$ of the sensor are equal to those in (9). The twin-T capacitance $C_{1}$ is taken equal to $0.24 \mu \mathrm{F}$, which means that $R_{1}=11.05 \mathrm{k} \Omega$. The capacitance $C_{1}$ cannot be taken too small. For a smaller $C_{1}, R_{1}$ becomes larger, since $C_{1}$ and $R_{1}$ are related to each other as follows,

$$
\frac{1}{2 \pi C_{1} R_{1}}=60 \mathrm{~Hz} \text {. }
$$

For a larger $R_{1}$, voltage output across the receiving impedance $R_{\mathrm{g}}$ becomes smaller. The voltage output across $R_{\mathrm{g}}$, which will be denoted by $V_{\mathrm{g}}$, is calculated, assuming that the amplitude of the source e.m.f. $E$ be equal to unity. The algebraic expression of the transfer function which relates $V_{\mathrm{g}}$ to $E$, is obtained, applying the theory of two-port circuit (e.g., LewIS and Pryce, 1965). We found it convenient to use the $F$ matrices of the sensor and of the twin-T filter. Refer to ZAMBRESKY (1977) for more details of the mathematical procedure. The voltage output $V_{\mathrm{g}}$ is plotted in Fig. 3 for several different values of $R_{\mathrm{g}}$. For the values larger than $45.4 \mathrm{k} \Omega$, a resonance peak appears at about $5 \mathrm{~Hz}$. The peak is higher for larger values of $R_{\mathrm{g}}$. Such a peak is undesirable. For the values of $R_{\mathrm{g}}$ less than $11.35 \mathrm{k} \Omega$, no peak appears; the response function gradually decreases for higher frequencies. The decrease is more gradual for lower values of $R_{\mathrm{g}}$. For $R_{\mathrm{g}}=22.7 \mathrm{k} \Omega$, the response curve is very similar to the case of the Butterworth filter. In every case, the response curve is greatly different from the ideal case that one would expect for twin-T filters. In the ideal case, the response curve in the frequency range of Fig. 3 that is of interest to us, would be virtually flat. The $-3 \mathrm{db}$ point of symmetric twin-T filters should exist between 20 and $30 \mathrm{~Hz}$. Out of the five cases shown in Fig. 3, the most desirable one is the case for $R_{\mathrm{g}}=22.7 \mathrm{k} \Omega$, and this case is similar to the Butterworth filter. In other words, the best that one can expect from the twin-T filter arrangement is practically nothing more than what one can obtain using the Butterworth filter.

\section{Concluding Remarks}

The Butterworth filter technique has actually been applied to one type of induction magnetometer designed and built at the University of British Columbia. Fifteen units of this type were built and used for observation of magnetic pulsations carried out simultaneously at five stations in Manitova in August and September of 1976. The technique has proved to be very useful and easy to apply in field work. 
The obtained data show that $60 \mathrm{~Hz}$ man-made noise was effectively rejected at every station without sacrificing Pc 1 signals, magnetic pulsations of the highest frequency range.

The authors sincerely thank Professor R. D. Russell, Professor T. Oguti, Dr. K. Hayashi, and Mr. H. Ueda for discussions. The authors are also indebted to the technical staff of the Department of Geophysics and Astronomy, University of British Columbia for assistance. This research was supported by the National Research Council of Canada under Grants A-3564 and E-2923.

\section{REFERENCES}

CAMPBell, W. H., Induction loop antennas for geomagnetic field variation measurements, Technical Report, ERL 123-ESL6, Environmental Science Services Administration, Boulder, Colorado, U.S.A., 1969.

Lewis, W. E. and D. G. Pryce, The Application of Matrix Theory to Electrical Engineering, pp. 141 171, E. \& F. N. Spon, Ltd., London, 1965.

UedA, H. and T. WATANABE, Several problems about sensitivity and frequency response of an induction magnetometer, Sci. Rep. Tohoku Univ., Ser. 5, Geophys., 22, 107-127, 1975a.

Ueda, H. and T. Watanabe, Comments on the anti-resonance method to measure the circuit constants of a coil used as a sensor of an induction magnetometer-Technical notes, Sci. Rep. Tohoku Univ., Ser. 5, Geophys., 22, 129-135, 1975b.

ZAMBRESKY, Liana F., The calibration of a portable induction magnetometer system, M.Sc. Thesis, University of British Columbia, 1977. 